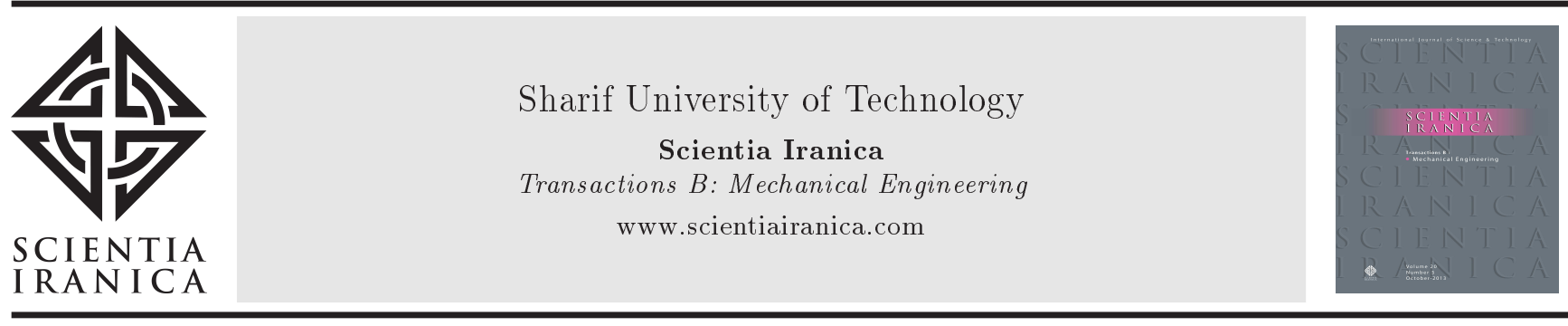

\title{
Application of Petrov-Galerkin finite element method to shallow water waves model: Modified Korteweg-de Vries equation
}

\author{
T. Ak ${ }^{a, *}$, S.B.G. Karakoc ${ }^{\mathrm{b}}$ and A. Biswas ${ }^{\mathrm{c}, \mathrm{d}}$ \\ a. Department of Transportation Engineering, Yalova University, 77100 Yalova, Turkey. \\ b. Department of Mathematics, Nevsehir Haci Bektas Veli University, 50300 Nevsehir, Turkey. \\ c. Department of Mathematical Sciences, Delaware State University, Dover, DE 19901-227r, USA. \\ d. Department of Mathematics, King Abdulaziz University, Jeddah-21589, Saudi Arabia. \\ Received 25 December 2015; received in revised form 28 April 2016; accepted 10 May 2016
}

\section{KEYWORDS \\ Modified KdV \\ equation; \\ Petrov-Galerkin \\ method; \\ Shallow water; \\ Solitary waves; \\ Soliton.}

\begin{abstract}
In this article, modified Korteweg-de Vries (mKdV) equation is solved numerically by using lumped Petrov-Galerkin approach, where weight functions are quadratic and the element shape functions are cubic B-splines. The proposed numerical scheme is tested by applying four test problems including single solitary wave, interaction of two and three solitary waves, and evolution of solitons with the Gaussian initial condition. In order to show the performance of the algorithm, the error norms, $L_{2}, L_{\infty}$, and a couple of conserved quantities are computed. For the linear stability analysis of numerical algorithm, Fourier method is also investigated. As a result, the computed results show that the presented numerical scheme is a successful numerical technique for solving the $\mathrm{mKdV}$ equation. Therefore, the presented method is preferable to some recent numerical methods.
\end{abstract}

(C) 2017 Sharif University of Technology. All rights reserved.

\section{Introduction}

The theory of nonlinear evolution equations (NLEEs) is a very popular and fascinating area of research in the field of applied mathematics and theoretical physics [19]. A few of the focused areas of research with NLEEs are fluid dynamics, nonlinear optics, nuclear physics, plasma physics, mathematical biosciences, and several others. This paper will focus on the numerical aspects of the dynamics of shallow water waves along lakes' shores and beaches modeled by a very popular NLEE. This is the modified Korteweg-de Vries $(\mathrm{mKdV})$ equation [10-21].

While several forms of numerical analyses were reported in the past, this paper addresses $\mathrm{mKdV}$

\footnotetext{
*. Corresponding author.

E-mail address: turgut.ak@yalova.edu.tr (T.Ak)
}

equation with a very popular and effective numerical scheme. This is the cubic B-spline Petrov-Galerkin method. The topics covered in this paper are linear stability analysis, the dynamics of single solitary wave of $\mathrm{mKdV}$ equation, interaction of two solitary waves and three solitary waves, and evolution of solitons. Several numerical examples are given to illustrate this powerful algorithm. In conclusion, the error analysis is also investigated in detail.

\section{Cubic B-spline Petrov-Galerkin method}

In this study, we will consider the modified Kortewegde Vries $(\mathrm{mKdV})$ equation:

$$
U_{t}+\varepsilon U^{2} U_{x}+\mu U_{x x x}=0,
$$

with the boundary conditions $U \rightarrow 0$ as $x \rightarrow \pm \infty$, where $\varepsilon$ and $\mu$ are positive parameters, and subscripts 
$x$ and $t$ denote the differentiation.

Let us consider the solution domain is limited to a finite interval $a \leq x \leq b$. The interval $[a, b]$ is divided into $N$ equal subinterval by the points $x_{m}$, such that $a=x_{0}<x_{1} \cdots<x_{N}=b$ and length $h=\frac{b-a}{N}=$ $\left(x_{m+1}-x_{m}\right), m=1,2, \cdots, N$. The mKdV equation (Eq. (1)) is considered with respect to the boundary conditions:

$$
\begin{aligned}
& U(a, t)=0, \quad U(b, t)=0, \\
& U_{x}(a, t)=0, \quad U_{x}(b, t)=0, \quad t>0,
\end{aligned}
$$

and the initial condition:

$$
U(x, 0)=f(x), \quad a \leq x \leq b,
$$

where $f(x)$ is a prescribed function. Physical boundary conditions require $U$ and $U_{x} \rightarrow 0$ that $U \rightarrow 0$ for $x \rightarrow \pm \infty$. The cubic B-spline functions $\phi_{m}(x)$, $m=-1(1) N+1$, are defined at the knots $x_{m}$ by the relationships [22]:

$$
\begin{aligned}
& \phi_{m}(x) \\
& =\frac{1}{h^{3}}\left\{\begin{array}{cc}
\left(x-x_{m-2}\right)^{3} & x \in\left[x_{m-2}, x_{m-1}\right], \\
h^{3}+3 h^{2}\left(x-x_{m-1}\right) & \\
+3 h\left(x-x_{m-1}\right)^{2} & x \in\left[x_{m-1}, x_{m}\right], \\
-3\left(x-x_{m-1}\right)^{3} & \\
h^{3}+3 h^{2}\left(x_{m+1}-x\right) & \\
-3\left(x_{m+1}-x\right)^{2} & x \in\left[x_{m}, x_{m+1}\right],(4) \\
\left(x_{m+2}-x\right)^{3}, & \\
0 & \text { otherwise. }
\end{array}\right.
\end{aligned}
$$

The set of functions $\left\{\phi_{-1}, \phi_{0}, \phi_{1}, \phi_{2}\right\}$ forms a basis for functions defined over the interval $a \leq x \leq b$. So, the numerical solution $U_{N}(x, t)$ to the exact solution $U_{N}(x, t)$ is given by:

$$
U_{N}(x, t)=\sum_{j=-1}^{N+1} \delta_{j}(t) \phi_{j}(x),
$$

where $\delta_{j}$ is time-dependent quantities to be determined from the boundary and weighted residual conditions. Each cubic B-spline covers four elements so that each element $\left[x_{m}, x_{m+1}\right]$ is covered by four splines. Applying a local coordinate system to typical finite element $\left[x_{m}, x_{m+1}\right]$ is defined by:

$$
h \eta=x-x_{m},
$$

such that $0 \leq \eta \leq 1$, the finite interval $\left[x_{m}, x_{m+1}\right]$ is converted to a more easily workable interval $[0,1]$. In this case, the cubic B-spline shape functions depending on variable $\eta$ over the element $[0,1]$ can be defined in the following form:

$$
\begin{aligned}
& \phi_{m-1}=(1-\eta)^{3}, \\
& \phi_{m}=1+3(1-\eta)+3(1-\eta)^{2}-3(1-\eta)^{3}, \\
& \phi_{m+1}=1+3 \eta+3 \eta^{2}-3 \eta^{3}, \\
& \phi_{m+2}=\eta^{3} .
\end{aligned}
$$

All splines, apart from $\phi_{m-1}(x), \phi_{m}(x), \phi_{m+1}(x)$, and $\phi_{m+2}(x)$, are null over the element $\left[x_{m}, x_{m+1}\right]$. Over the typical element $\left[x_{m}, x_{m+1}\right]$, the numerical solution $U_{N}(x, t)$ is given by:

$$
U_{N}(x, t)=\sum_{j=m-1}^{m+2} \phi_{j}(x) \delta_{j}(t)
$$

where $\delta_{m-1}, \delta_{m}, \delta_{m+1}$, and $\delta_{m+2}$ act as element parameters and B-splines $\phi_{m-1}, \phi_{m}, \phi_{m+1}$, and $\phi_{m+2}$ as element shape functions. The values of $\phi_{j}(x)$ and its derivative may be tabulated as in Table 1.

Using cubic B-splines (Eq. (7)) and trial function (Eq. (8)), the nodal values of $U, U^{\prime}$, and $U^{\prime \prime}$ at the knot $x_{m}$ are given in terms of the element parameters $\delta_{m}$ by:

$$
\begin{aligned}
& U_{m}=U\left(x_{m}\right)=\delta_{m-1}+4 \delta_{m}+\delta_{m+1}, \\
& U_{m}^{\prime}=U^{\prime}\left(x_{m}\right)=\frac{3}{h}\left(-\delta_{m-1}+\delta_{m+1}\right), \\
& U_{m}^{\prime \prime}=U^{\prime \prime}\left(x_{m}\right)=\frac{6}{h^{2}}\left(\delta_{m-1}-2 \delta_{m}+\delta_{m+1}\right),
\end{aligned}
$$

where the symbols' and " denote the first and second differentiations with respect to $x$, respectively. The splines $\phi_{m}(x)$ and their two principle derivatives vanish outside the interval $\left[x_{m-2}, x_{m+2}\right]$. The weight function $\psi_{m}$ is taken a quadratic B-spline. Quadratic B-spline $\psi_{m}$ at the knots $x_{m}$ is defined over the interval $[a, b]$ by:

Table 1. Cubic B-splines and its derivatives at nodes $x_{m}$.

\begin{tabular}{cccccc}
$\boldsymbol{x}$ & $\boldsymbol{x}_{\boldsymbol{m}-\mathbf{2}}$ & $\boldsymbol{x}_{\boldsymbol{m}-\mathbf{1}}$ & $\boldsymbol{x}_{\boldsymbol{m}}$ & $\boldsymbol{x}_{\boldsymbol{m}+\mathbf{1}}$ & $\boldsymbol{x}_{\boldsymbol{m}+\mathbf{2}}$ \\
\hline$\phi_{m}(x)$ & 0 & 1 & 4 & 1 & 0 \\
$\phi_{m}^{\prime}(x)$ & 0 & $\frac{3}{h}$ & 0 & $-\frac{3}{h}$ & 0 \\
$\phi_{m}^{\prime \prime}(x)$ & 0 & $\frac{6}{h^{2}}$ & $-\frac{12}{h^{2}}$ & $\frac{6}{h^{2}}$ & 0 \\
\hline
\end{tabular}




$$
\psi_{m}(x)=\frac{1}{h^{2}}\left\{\begin{array}{cc}
\left(x_{m+2}-x\right)^{2} & \\
-3\left(x_{m+1}-x\right)^{2} & {\left[x_{m-1}, x_{m}\right],} \\
+3\left(x_{m}-x\right)^{2} & \\
\left(x_{m+2}-x\right)^{2} & {\left[x_{m}, x_{m+1}\right]} \\
-3\left(x_{m+1}-x\right)^{2} & \\
\left(x_{m+2}-x\right)^{2} & {\left[x_{m+1}, x_{m+2}\right]} \\
0 & \text { otherwise. }
\end{array}\right.
$$

Using the local coordinate transformation for the finite element $\left[x_{m}, x_{m+1}\right]$ by $h \eta=x-x_{m}, 0 \leq \eta \leq 1$, quadratic B-spline $\psi_{m}$ can be defined as:

$$
\begin{aligned}
& \psi_{m-1}=(1-\eta)^{2}, \\
& \psi_{m}=1+2 \eta-2 \eta^{2}, \\
& \psi_{m+1}=\eta^{2} .
\end{aligned}
$$

Applying the Petrov-Galerkin approach to Eq. (1), we obtain the weak form of Eq. (1):

$$
\int_{0}^{1} \psi\left(U_{t}+\varepsilon U^{2} U_{x}+\mu U_{x x x}\right) d x=0 .
$$

For a single element $\left[x_{m}, x_{m+1}\right]$, using transformation in Eq. (6) into Eq. (12), we obtain:

$$
\int_{0}^{1} \psi\left(U_{t}+\varepsilon\left(\frac{U^{2}}{h}\right) U_{\eta}+\mu\left(\frac{1}{h^{3}}\right) U_{\eta \eta \eta}\right) d \eta=0
$$

Integrating Eq. (13) by parts and using Eq. (1) lead to:

$$
\left.\int_{0}^{1}\left[\psi\left(U_{t}+\lambda U_{\eta}\right)+\xi \Psi_{\eta} U_{\eta t}\right)\right] d \eta=\left.\xi \Psi U_{\eta t}\right|_{0} ^{1}
$$

where $\lambda=\frac{U^{2}}{h}$ and $\xi=\frac{\mu}{h^{3}}$ Taking the weight function, $\Psi_{i}$, with quadratic B-spline shape functions given by Eq. (11) and substituting approximation in Eq. (8) into integral Eq. (14), we obtain the element contributions in the form:

$$
\begin{gathered}
\sum_{j=m-1}^{m+2}\left[\int_{0}^{1} \psi_{i} \phi_{j} d \eta\right] \dot{\delta}_{j}^{e}+\sum_{j=m-1}^{m+2}\left[\left(\varepsilon \lambda \int_{0}^{1} \psi_{i} \phi_{j}^{\prime} d \eta\right)\right. \\
\left.-\left(\xi \int_{0}^{1} \psi_{i}^{\prime} \phi_{j}^{\prime \prime} d \eta\right)+\left(\left.\xi \psi_{i} \phi_{j}^{\prime \prime}\right|_{0} ^{1}\right)\right] \delta_{j}^{e}=0,
\end{gathered}
$$

which can be written in a matrix form as follows:

$$
\left[A^{e}\right] \dot{\delta}^{e}+\left[\varepsilon \lambda B^{e}-\xi\left(C^{e}-D^{e}\right)\right] \delta^{e}=0,
$$

where $\delta^{e}=\left(\delta_{m-1}, \delta_{m}, \delta_{m+1}, \delta_{m+2}\right)^{T}$ are the element parameters, and the dot denotes differentiation with respect to $t$. The element matrices $A^{e}, B^{e}, C^{e}$, and $D^{e}$ are rectangular $3 \times 4$ given by the following integrals:

$$
\begin{aligned}
& A_{i j}^{e}=\int_{0}^{1} \psi_{i} \phi_{j} d \eta=\frac{1}{60}\left[\begin{array}{cccc}
10 & 71 & 38 & 1 \\
19 & 221 & 221 & 19 \\
1 & 38 & 71 & 10
\end{array}\right], \\
& B_{i j}^{e}=\int_{0}^{1} \psi_{i} \phi_{j}^{\prime} d \eta=\frac{1}{10}\left[\begin{array}{cccc}
-6 & -7 & 12 & 1 \\
-13 & -41 & 41 & 13 \\
-1 & -12 & 7 & 6
\end{array}\right], \\
& C_{i j}^{e}=\int_{0}^{1} \psi_{i}^{\prime} \phi_{j}^{\prime \prime} d \eta=\left[\begin{array}{cccc}
-4 & 6 & 0 & -2 \\
2 & -6 & 6 & -2 \\
2 & 0 & -6 & 4
\end{array}\right], \\
& D_{i j}^{e}=\left.\psi_{i} \phi_{j}^{\prime \prime}\right|_{0} ^{1}=\left[\begin{array}{cccc}
-6 & 12 & -6 & 0 \\
-6 & 18 & -18 & 6 \\
0 & 6 & -12 & 6
\end{array}\right],
\end{aligned}
$$

where $i$ takes only the values 1,2 , and $3 ; j$ takes only the values $m-1, m, m+1$, and $m+2$ for the typical element $\left[x_{m}, x_{m+1}\right]$. A lumped value for $\lambda$ is found from $\frac{1}{4}\left(U_{m}+U_{m+1}\right)^{2}$ as:

$$
\lambda=\frac{1}{4 h}\left(\delta_{m-1}+5 \delta_{m}+5 \delta_{m+1}+\delta_{m+2}\right)^{2} .
$$

By considering together contributions from all elements, the following matrix equation becomes:

$$
[A] \dot{\delta}+[\varepsilon \lambda B-\xi(C-D)] \delta=0,
$$

where $\delta=\left(\delta_{-1}, \delta_{0}, \cdots, \delta_{N}, \delta_{N+1}\right)^{T}$ is a global element parameter. The matrices $A, B$, and $\lambda D$ are rectangular and $m$ of each row has the following form:

$$
\begin{aligned}
& A=\frac{1}{60}(1,57,302,302,57,1), \\
& \lambda B=\frac{1}{10}\left(-\lambda_{1},-12 \lambda_{1}-13 \lambda_{2}, 7 \lambda_{1}-41 \lambda_{2}\right. \\
& \left.-6 \lambda_{3}, 6 \lambda_{1}+41 \lambda_{2}-7 \lambda_{3}, 13 \lambda_{2}+12 \lambda_{3}, \lambda_{3}\right), \\
& C=2(1,1,-8,8,-1,-1), \\
& D=(0,0,0,0,0,0,0)
\end{aligned}
$$

where:

$$
\begin{aligned}
& \lambda_{1}=\frac{1}{4 h}\left(\delta_{m-2}+5 \delta_{m-1}+5 \delta_{m}+\delta_{m+1}\right)^{2}, \\
& \lambda_{2}=\frac{1}{4 h}\left(\delta_{m-1}+5 \delta_{m}+5 \delta_{m+1}+\delta_{m+2}\right)^{2}, \\
& \lambda_{3}=\frac{1}{4 h}\left(\delta_{m}+5 \delta_{m+1}+5 \delta_{m+2}+\delta_{m+3}\right)^{2} .
\end{aligned}
$$


Substituting the Crank-Nicolson approach, $\delta=\frac{1}{2}\left(\delta^{n}+\right.$ $\left.\delta^{n+1}\right)$, and the forward finite difference, $\dot{\delta}=\frac{\delta^{n+1}-\delta^{n}}{\Delta t}$, in Eq. (18), we obtain the following matrix system:

$$
\begin{aligned}
\{A & \left.+[\varepsilon \lambda B-\xi(C-D)] \frac{\Delta t}{2}\right\} \delta^{n+1} \\
& =\left\{A-[\varepsilon \lambda B-\xi(C-D)] \frac{\Delta t}{2}\right\} \delta^{n},
\end{aligned}
$$

where $\Delta t$ is the time step. Applying the boundary condition (2) to the system (19), we make the matrix equation square. The resulting system can be efficiently solved by a variant of the Thomas algorithm. Two or three inner iterations are applied to $\delta^{n *}=$ $\delta^{n}+\frac{1}{2}\left(\delta^{n}-\delta^{n-1}\right)$ at each time in order to improve the accuracy. A typical member of the matrix system (19) may be written in terms of the nodal parameters $\delta^{n}$ and $\delta^{n+1}$ as:

$$
\begin{aligned}
\gamma_{1} \delta_{m-2}^{n+1} & +\gamma_{2} \delta_{m-1}^{n+1}+\gamma_{3} \delta_{m}^{n+1}+\gamma_{4} \delta_{m+1}^{n+1}+\gamma_{5} \delta_{m+2}^{n+1} \\
& +\gamma_{6} \delta_{m+3}^{n+1}=\gamma_{6} \delta_{m-2}^{n}+\gamma_{5} \delta_{m-1}^{n}+\gamma_{4} \delta_{m}^{n} \\
& +\gamma_{3} \delta_{m+1}^{n}+\gamma_{2} \delta_{m+2}^{n}+\gamma_{1} \delta_{m+3}^{n}
\end{aligned}
$$

where:

$$
\begin{aligned}
& \gamma_{1}=\frac{1}{60}-\frac{\varepsilon \lambda \Delta t}{20}-\xi \Delta t, \\
& \gamma_{2}=\frac{57}{60}-\frac{25 \varepsilon \lambda \Delta t}{20}-\xi \Delta t, \\
& \gamma_{3}=\frac{302}{60}-\frac{40 \varepsilon \lambda \Delta t}{20}+8 \xi \Delta t, \\
& \gamma_{4}=\frac{302}{60}+\frac{40 \varepsilon \lambda \Delta t}{20}-8 \xi \Delta t, \\
& \gamma_{5}=\frac{57}{60}+\frac{25 \varepsilon \lambda \Delta t}{20}+\xi \Delta t, \\
& \gamma_{6}=\frac{1}{60}+\frac{\varepsilon \lambda \Delta t}{20}+\xi \Delta t,
\end{aligned}
$$

which all depend on $\delta^{n}$.

To evaluate the vector parameters, $\delta^{n}$, the initial vector $\delta^{0}$ must be determined from the initial and boundary conditions. So, the approximation in Eq. (8) can be rewritten for the initial condition:

$$
U_{N}(x, 0)=\sum_{m=-1}^{N+1} \phi_{m}(x) \delta_{m}^{0}
$$

where the parameters $\delta_{m}^{0}$ will be determined. Using relations at the knots:

$$
\begin{aligned}
& U_{N}\left(x_{m}, 0\right)=U\left(x_{m}, 0\right), \\
& U_{N}^{\prime}\left(x_{0}, 0\right)=U^{\prime}\left(x_{N}, 0\right)=0, \quad m=0,1, \cdots, N,
\end{aligned}
$$

together with derivative condition, the initial vector $\delta^{0}$ can be determined from the following matrix equation:

$$
\left[\begin{array}{ccccccc}
-3 & 0 & 3 & & & & \\
1 & 4 & 1 & & & & \\
& & & \ddots & & & \\
& & & & 1 & 4 & 1 \\
& & & & -3 & 0 & 3
\end{array}\right]\left[\begin{array}{c}
\delta_{-1}^{0} \\
\delta_{0}^{0} \\
\vdots \\
\delta_{N}^{0} \\
\delta_{N+1}^{0}
\end{array}\right]=\left[\begin{array}{c}
U^{\prime}\left(x_{0,0}\right) \\
U\left(x_{0,0}\right) \\
\vdots \\
U\left(x_{N, 0}\right) \\
U^{\prime}\left(x_{N, 0}\right)
\end{array}\right]
$$

which can be solved using a variant of the Thomas algorithm.

\section{Stability analysis}

For the linear stability analysis of the numerical algorithm, we use the Fourier method and assume that the quantity $U$ in the non-linear term $U^{2} U_{x}$ is locally constant. In this case, the $\mathrm{mKdV}$ equation can be linearized. The growth factor $\rho$ of the error in a typical mode of amplitude,

$$
\delta_{m}^{n}=\rho^{n} e^{i j k h}
$$

where $k$ is the mode number, and $h$ is the element size. Substituting the Fourier mode (Eq. (23)) into Eq. (20) gives the growth factor, $\rho$, of the form:

$$
\rho=\frac{a-i b}{a+i b}
$$

where:

$$
\begin{aligned}
a= & 302 \cos \left(\frac{k h}{2}\right)+57 \cos \left(\frac{3 k h}{2}\right)+\cos \left(\frac{5 k h}{2}\right), \\
b= & {[(120 \varepsilon \lambda-480 \xi) \Delta t] \sin \left(\frac{k h}{2}\right) } \\
& +[(75 \varepsilon \lambda+60 \xi) \Delta t] \sin \left(\frac{3 k h}{2}\right) \\
& +[(3 \varepsilon \lambda+60 \xi) \Delta t] \sin \left(\frac{5 k h}{2}\right) .
\end{aligned}
$$

The modulus of $|\rho|$ is 1 ; therefore, the linearized scheme is unconditionally stable.

\section{Numerical examples and results}

Numerical results of the $\mathrm{mKdV}$ equation are obtained for three problems: the motion of a single-solitary wave, interaction of two and three solitary waves. We use the error norm $L_{2}$ as in the following:

$$
L_{2}=\left\|U^{\text {exact }}-U_{N}\right\|_{2} \simeq \sqrt{h \sum_{J=1}^{N}\left|U_{j}^{\text {exact }}-\left(U_{N}\right)_{j}\right|^{2}}
$$


and the error norm $L_{\infty}$ as in:

$$
\begin{aligned}
L_{\infty} & =\left\|U^{\text {exact }}-U_{N}\right\|_{\infty} \simeq \max _{j}\left|U_{j}^{\text {exact }}-\left(U_{N}\right)_{j}\right| \\
j & =1,2, \cdots, N-1,
\end{aligned}
$$

so that we can calculate the difference between analytical and numerical solutions at some specified times. Three of the many infinitely conserved quantities of mKdV Eq. (1) are:

$$
\begin{aligned}
I_{1} & =\int_{a}^{b} U d x \simeq h \sum_{J=1}^{N} U_{j}^{n}, \\
I_{2} & =\int_{a}^{b} U^{2} d x \simeq h \sum_{J=1}^{N}\left(U_{j}^{n}\right)^{2}, \\
I_{3} & =\int_{a}^{b}\left[U^{4}-\frac{6 \mu}{\varepsilon}\left(U_{x}\right)^{2}\right] d x \\
& \simeq h \sum_{J=1}^{N}\left[\left(U_{j}^{n}\right)^{4}-\frac{6 \mu}{\varepsilon}\left(U_{x}\right)_{j}^{n}\right],
\end{aligned}
$$

which correspond to conversation of mass, momentum, and energy, respectively [23,24]. In the simulation of solitary wave motion, the invariants $I_{1}, I_{2}$, and $I_{3}$ are monitored to check the conversation of the numerical algorithm.

\subsection{The motion of single solitary wave}

As the first problem, Eq. (1) is considered with respect to the boundary conditions $U \rightarrow 0$ as $x \rightarrow \pm \infty$ and the initial condition:

$$
U(x, 0)=\sqrt{\frac{6 c}{\varepsilon}} \operatorname{sech}\left[\sqrt{\frac{c}{\mu}}\left(x-x_{0}\right)\right] .
$$

The analytical solution of the mKdV can be written as:

$$
U(x, t)=\sqrt{\frac{6 c}{\varepsilon}} \operatorname{sech}\left[\sqrt{\frac{c}{\mu}}\left(x-c t-x_{0}\right)\right],
$$

where $\varepsilon, \mu, c$, and $x_{0}$ are arbitrary constants. The conserved quantities of motion for a solitary wave of amplitude $\sqrt{\frac{6 c}{\varepsilon}}$ and width depending on $\sqrt{\frac{c}{\mu}}$ may be evaluated analytically as in [13]:

$$
\begin{aligned}
& I_{1}=\pi \sqrt{\frac{6 \mu}{\varepsilon}}, \quad I_{2}=\frac{12 \sqrt{\mu c}}{\varepsilon}, \\
& I_{3}=-\frac{64 c^{2}}{\varepsilon^{2}} \sqrt{\frac{\mu}{c}} .
\end{aligned}
$$

The values of the parameters $\varepsilon=3, \mu=1, c=0.845$, $h=0.1$, and $\Delta t=0.01$ are chosen over the spatial interval $[0,80]$ to coincide with that of [13]. For these parameters, the solitary wave has an amplitude $A=$ $\sqrt{\frac{6 c}{\varepsilon}}=1.3$. Numerical computations are done up to time $t=20$. The obtained results are tabulated in Table 2. The conserved quantities and error norms $L_{2}$ and $L_{\infty}$ are shown at selected times. Table 2 represents a comparison of the values of the invariants and error norms obtained by the present method with those obtained by other method [13]. It is clearly seen from the table that the invariants remain almost constant during the computer run. It is observed from the table that the error norms obtained by our method are smaller than those given in [13]. In Figure 1, the numerical solutions are displayed at $t=0,1,2, \cdots, 20$. As seen from the figure, the soliton moves to the right at a constant speed and almost unchanged amplitude as time increases, as expected. In Figure 2, the motion of single soliton is plotted with $\varepsilon=3, \mu=1, c=0.845$, $h=0.1$, and $\Delta t=0.01$ at selected times from $t=0$ to $t=20$. Errors distributions at time $t=20$ are depicted for solitary waves amplitudes 1.3 in Figure 3 to show the errors between the analytical and numerical results over the problem domain.

Table 2. Invariants and error norms for single solitary wave with $\varepsilon=3, \mu=1, c=0.845, h=0.1$, and $\Delta t=0.01$, $0 \leq x \leq 80$.

\begin{tabular}{ccccccc}
\hline Results & $\boldsymbol{t}$ & $\mathbf{1}$ & $\mathbf{5}$ & $\mathbf{1 0}$ & $\mathbf{1 5}$ & $\mathbf{2 0}$ \\
\hline \multirow{2}{*}{$I_{1}$} & Present & 4.442866 & 4.442866 & 4.442866 & 4.442866 & 4.442866 \\
& {$[13]$} & 4.443000 & 4.443138 & 4.444142 & 4.443420 & 4.443171 \\
\hline \multirow{2}{*}{$I_{2}$} & Present & 3.676941 & 3.676941 & 3.676941 & 3.676941 & 3.676941 \\
& {$[13]$} & 3.677069 & 3.677535 & 3.678094 & 3.678642 & 3.679192 \\
\hline \multirow{2}{*}{$I_{3}$} & Present & 2.072795 & 2.073537 & 2.073699 & 2.073776 & 2.073846 \\
& {$[13]$} & 2.073575 & 2.074357 & 2.075303 & 2.076232 & 2.077161 \\
\hline \multirow{2}{*}{$L_{2}$-Error } & Present & $6.286951 \mathrm{e}-04$ & $1.249516 \mathrm{e}-03$ & $2.131860 \mathrm{e}-03$ & $2.949376 \mathrm{e}-03$ & $3.641638 \mathrm{e}-03$ \\
& {$[13]$} & - & - & - & - & - \\
\hline \multirow{2}{*}{$L_{\infty}$-Error } & Present & $3.630992 \mathrm{e}-04$ & $8.397466 \mathrm{e}-04$ & $1.399503 \mathrm{e}-03$ & $1.880855 \mathrm{e}-03$ & $2.285638 \mathrm{e}-03$ \\
& {$[13]$} & $1.206756 \mathrm{e}-03$ & $3.621519 \mathrm{e}-03$ & $5.942047 \mathrm{e}-03$ & $7.626772 \mathrm{e}-03$ & $8.642137 \mathrm{e}-03$ \\
\hline
\end{tabular}




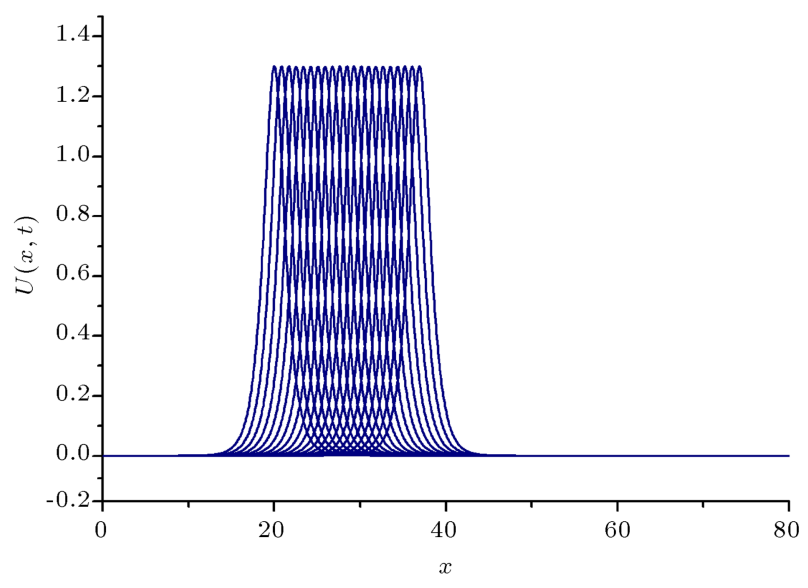

Figure 1. Single solitary wave with $\varepsilon=3, \mu=1$, $c=0.845, h=0.1, \Delta t=0.010$, and $0 \leq x \leq 80$ at $t=0,1,2, \cdots, 20$.
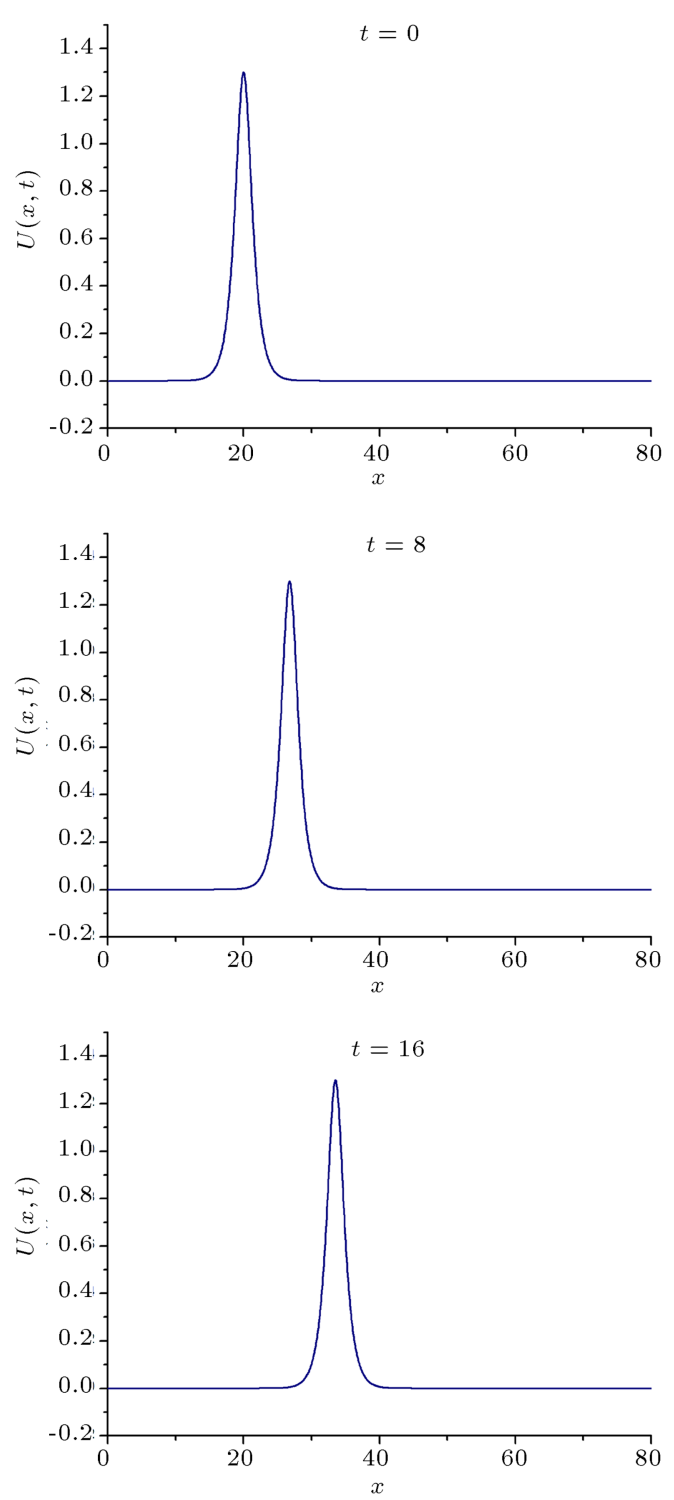

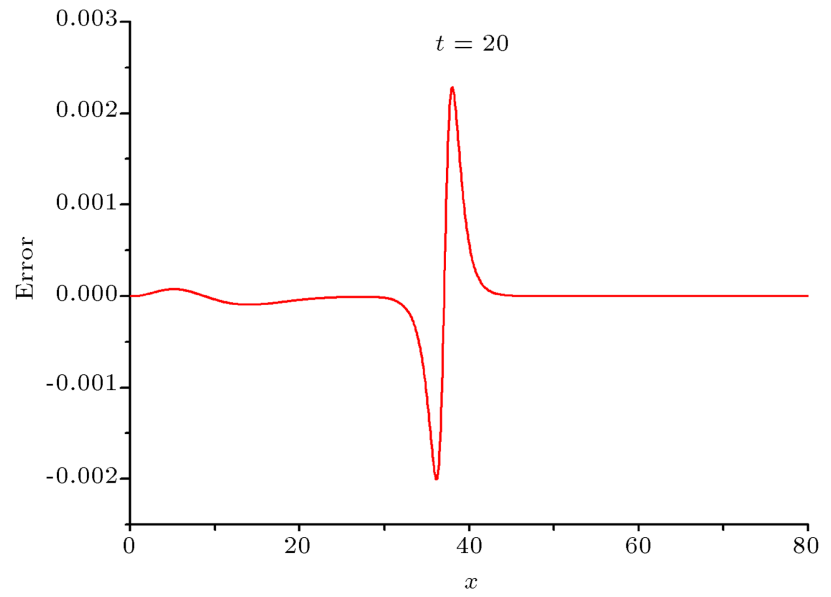

Figure 3. Error with $\varepsilon=3, \mu=1, c=0.845, h=0.1$, and $\Delta t=0.01,0 \leq x \leq 80, t=20$.
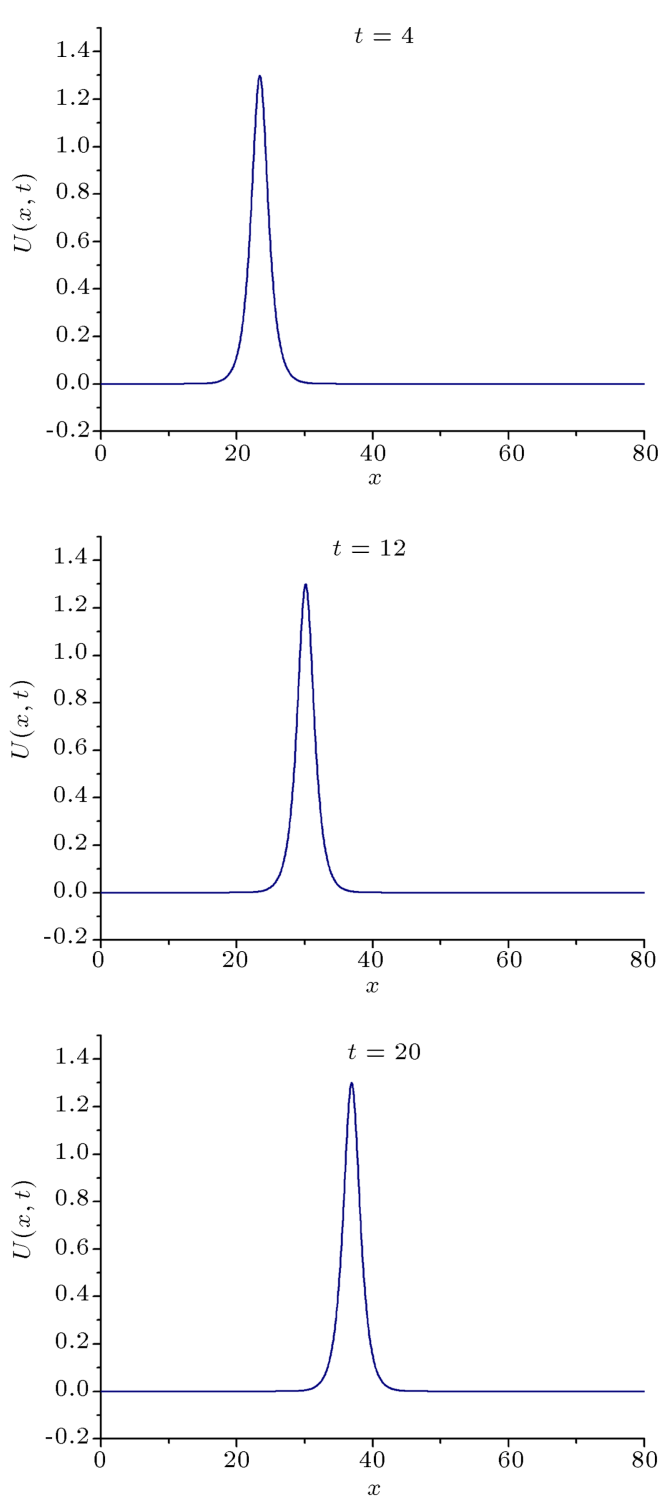

Figure 2. Single solitary wave with $\varepsilon=3, \mu=1, c=0.845, h=0.1, \Delta t=0.010$, and $0 \leq x \leq 80$ at $t=0,4,8,12,16$ and 20. 


\subsection{Interaction of two solitary waves}

As a second problem, we consider the interaction of two solitary waves by using the initial condition given by the linear sum of the two well-separated solitary waves having various amplitudes:

$$
U(x, 0)=\sum_{i=1}^{2} \alpha_{i} \operatorname{sech}\left[\beta_{i}\left(x-x_{i}\right)\right],
$$

where $\alpha_{i}=\sqrt{\frac{6 c_{i}}{\varepsilon}}, \beta_{i}=\sqrt{\frac{c_{i}}{\mu}}, i=1,2$, and $c_{i}$ and $x_{i}$ are arbitrary constants.

For the simulation, the parameters $\varepsilon=3, \mu=1$, $h=0.1, \Delta t=0.01, c_{1}=2, c_{2}=1, x_{1}=15$, and $x_{2}=25$ are chosen over the range $0 \leq x \leq 80$ to coincide with Ref. [13]. The experiment is run from $t=$ 0 to $t=20$ and the calculated values of the invariants $I_{1}, I_{2}$, and $I_{3}$ obtained by the present method with those obtained in [13] are compared in Table 3 . It is seen that the obtained values of the invariants remain almost constant during the computer run.

Figure 4 shows the development of the interaction of two solitary waves. It is clear from the figure that at $t=0$, the greater soliton is at the left position of the smaller soliton in the beginning of the run. With the increase in time, the greater soliton catches up the smaller one until at time $t=7$, when the smaller soliton is absorbed. The overlapping process continues until $t=8$, while greater soliton has overtaken the smaller soliton and gotten in the process of the separating. At time $t=16$, the interaction is complete, and the greater soliton has been separated completely.

\subsection{Interaction of three solitary waves}

As for the third problem, we study the behavior of the interaction of three solitary waves with different amplitudes and traveling in the same direction. So, we consider Eq. (1) with initial condition given by the linear sum of three well-separated solitary waves of different amplitudes:

$$
U(x, 0)=\sum_{i=1}^{3} \alpha_{i} \operatorname{sech}\left[\beta_{i}\left(x-x_{i}\right)\right]
$$

where $\alpha_{j}=\sqrt{\frac{6 c_{i}}{\varepsilon}}, \beta_{i}=\sqrt{\frac{c_{i}}{\mu}}, i=1,2,3, c_{i}$, and $x_{i}$ are arbitrary constants.

For the computational work, parameters $\varepsilon=3$, $\mu=1, h=0.1, \Delta t=0.01, c_{1}=2, c_{2}=1, c_{3}=0.5$, $x_{1}=15, x_{2}=25$, and $x_{3}=35$ are taken over the interval $0 \leq x \leq 80$. Simulations are done up to time $t=20$. Table 4 displays a comparison of the values of the invariants obtained by the present method with those obtained in [13]. It is seen from the table that the obtained values of the invariants remain

Table 3. Comparison of invariants for the interaction of two solitary waves with $\varepsilon=3, \mu=1, h=0.1, \Delta t=0.01, c_{1}=2$, $c_{2}=1, x_{1}=15$, and $x_{2}=25,0 \leq x \leq 80$.

\begin{tabular}{ccccccc}
\hline $\boldsymbol{I}$ & $\boldsymbol{t}$ & $\mathbf{1}$ & $\mathbf{5}$ & $\mathbf{1 0}$ & $\mathbf{1 5}$ & $\mathbf{2 0}$ \\
\hline \multirow{2}{*}{$I_{1}$} & Present & 8.885732 & 8.885732 & 8.885732 & 8.885732 & 8.885732 \\
& {$[13]$} & 8.886014 & 8.886776 & 8.889742 & 8.885983 & 8.884880 \\
\hline \multirow{2}{*}{$I_{2}$} & Present & 9.659345 & 9.659345 & 9.659345 & 9.659345 & 9.659345 \\
& {$[13]$} & 9.659527 & 9.663714 & 9.662547 & 9.661071 & 9.661224 \\
\hline \multirow{2}{*}{$I_{3}$} & Present & 10.270908 & 10.853235 & 10.954396 & 10.307195 & 10.338415 \\
& {$[13]$} & 10.239870 & 10.249000 & 10.246790 & 10.242580 & 10.242030 \\
\hline
\end{tabular}

Table 4. Comparison of invariants for the interaction of three solitary waves with $\varepsilon=3, \mu=1, h=0.1, \Delta t=0.01, c_{1}=2$, $c_{2}=1, c_{3}=0.5, x_{1}=15, x_{2}=25$, and $x_{3}=35,0 \leq x \leq 80$.

\begin{tabular}{ccccccc}
\hline $\boldsymbol{I}$ & $\boldsymbol{t}$ & $\mathbf{1}$ & $\mathbf{5}$ & $\mathbf{1 0}$ & $\mathbf{1 5}$ & $\mathbf{2 0}$ \\
\hline \multirow{2}{I_{1}}{} & Present & 13.328677 & 13.328677 & 13.328677 & 13.328677 & 13.328677 \\
& {$[13]$} & 13.329060 & 13.330630 & 13.338780 & 13.332640 & 13.332060 \\
\hline \multirow{2}{*}{$I_{2}$} & Present & 12.519943 & 12.519943 & 12.519943 & 12.519943 & 12.519943 \\
& {$[13]$} & 12.520280 & 12.526260 & 12.540860 & 12.526660 & 12.524900 \\
\hline & Present & 11.321264 & 12.452085 & 12.476293 & 12.413843 & 11.499239 \\
$I_{3}$ & {$[13]$} & 11.249790 & 11.261270 & 11.288040 & 11.259970 & 11.256730 \\
\hline
\end{tabular}



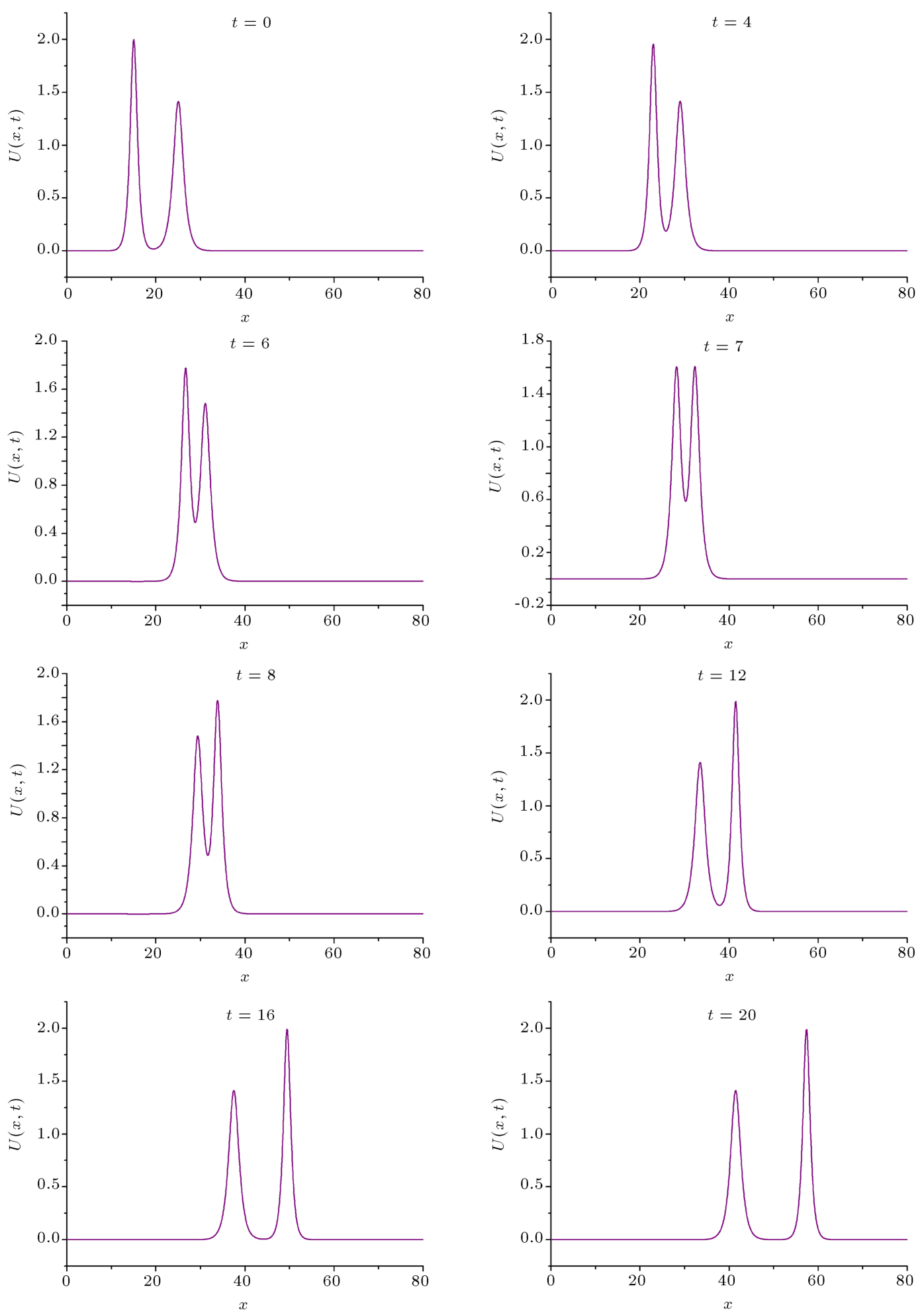

Figure 4. Interaction of two solitary waves with $\varepsilon=3, \mu=1, h=0.1, \Delta t=0.01, c_{1}=2, c_{2}=1, x_{1}=15, x_{2}=25$, and $0 \leq x \leq 80$ at $t=0,4,6,7,8,12,16$, and 20 .

almost constant during the computer run. As seen from Figure 5, interaction started about time $t=6$, overlapping processes occurred between time $t=6$ and $t=20$, and waves started to resume their original shapes after the time $t=20$.

\section{Evolution of solitons}

As for our last problem, evolution of the solitons has been studied using the Gaussian initial condition:

$$
U(x, 0)=\exp \left(-x^{2}\right),
$$



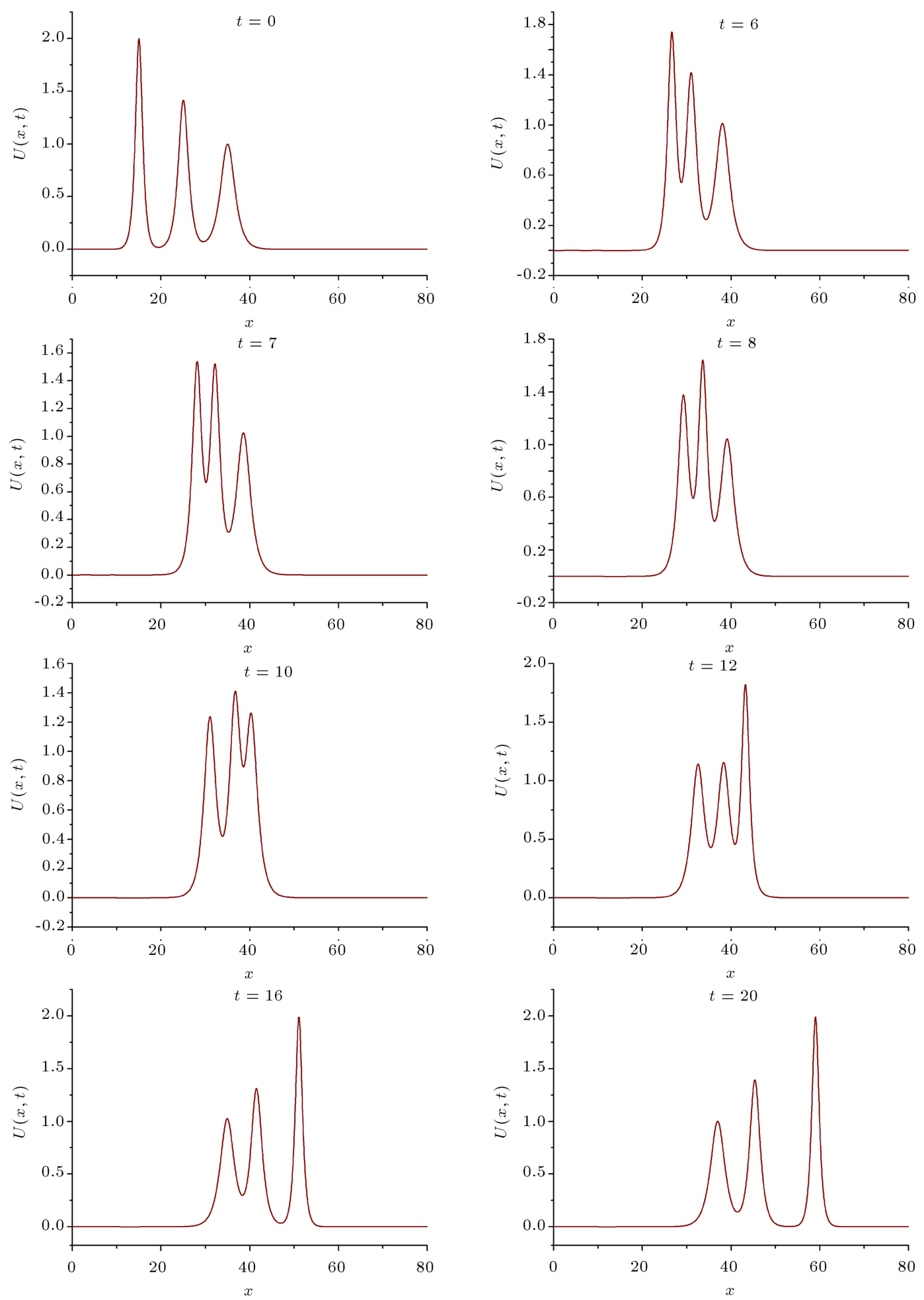

Figure 5. Interaction of three solitary waves with $\varepsilon=3, \mu=1, h=0.1, \Delta t=0.01, c_{1}=2, c_{2}=1, c_{3}=0.5, x_{1}=15$, $x_{2}=25, x_{3}=35$, and $0 \leq x \leq 80$ at $t=0,6,7,8,10,12,16$, and 20 .

for various values of $\mu$. In this case, the behavior of the solution depends on the values of $\mu$. Therefore, the values of $\mu=0.1$ and $\mu=0.04$ are chosen at the region of the $-50 \leq x \leq 50$. The numerical computations are done up to $t=10$. The values of the three invariants of motion for different $\mu$ are presented in Table 5 .
Also, Figures 6 and 7 illustrate the development of the Gaussian initial condition into solitary waves.

\section{Conclusion}

In this paper, a numerical treatment of the $\mathrm{mKdV}$ 
Table 5. Invariants for Gaussian initial condition with $\mu=0.1$ and $\mu=0.04, h=0.1, \Delta t=0.01$, and $-50 \leq x \leq 50$ at $0 \leq t \leq 10$.

\begin{tabular}{ccccccccc}
\hline \multirow{2}{*}{$\boldsymbol{t}$} & \multicolumn{3}{c}{$\boldsymbol{\mu}=\mathbf{0 . 1}$} & & \multicolumn{3}{c}{$\boldsymbol{\mu}=\mathbf{0 . 0 4}$} \\
\cline { 2 - 4 } \cline { 6 - 8 } & $\boldsymbol{I}_{\mathbf{1}}$ & $\boldsymbol{I}_{\mathbf{2}}$ & $\boldsymbol{I}_{\mathbf{3}}$ & & $\boldsymbol{I}_{\mathbf{1}}$ & $\boldsymbol{I}_{\mathbf{2}}$ & $\boldsymbol{I}_{\mathbf{3}}$ \\
\hline 0 & 1.772454 & 1.253314 & .2355683 & & 1.772454 & 1.253314 & 0.1958645 \\
2 & 1.772454 & 1.253314 & .2456447 & & 1.772454 & 1.253314 & 0.1845277 \\
4 & 1.772454 & 1.253314 & .2099601 & & 1.772454 & 1.253314 & 0.1912904 \\
6 & 1.772454 & 1.253314 & .2066328 & & 1.772454 & 1.253314 & 0.2079808 \\
8 & 1.772454 & 1.253314 & .2010120 & & 1.772454 & 1.253314 & 0.2229795 \\
10 & 1.772454 & 1.253314 & .1747070 & & 1.772454 & 1.253314 & 0.2363841 \\
\hline
\end{tabular}
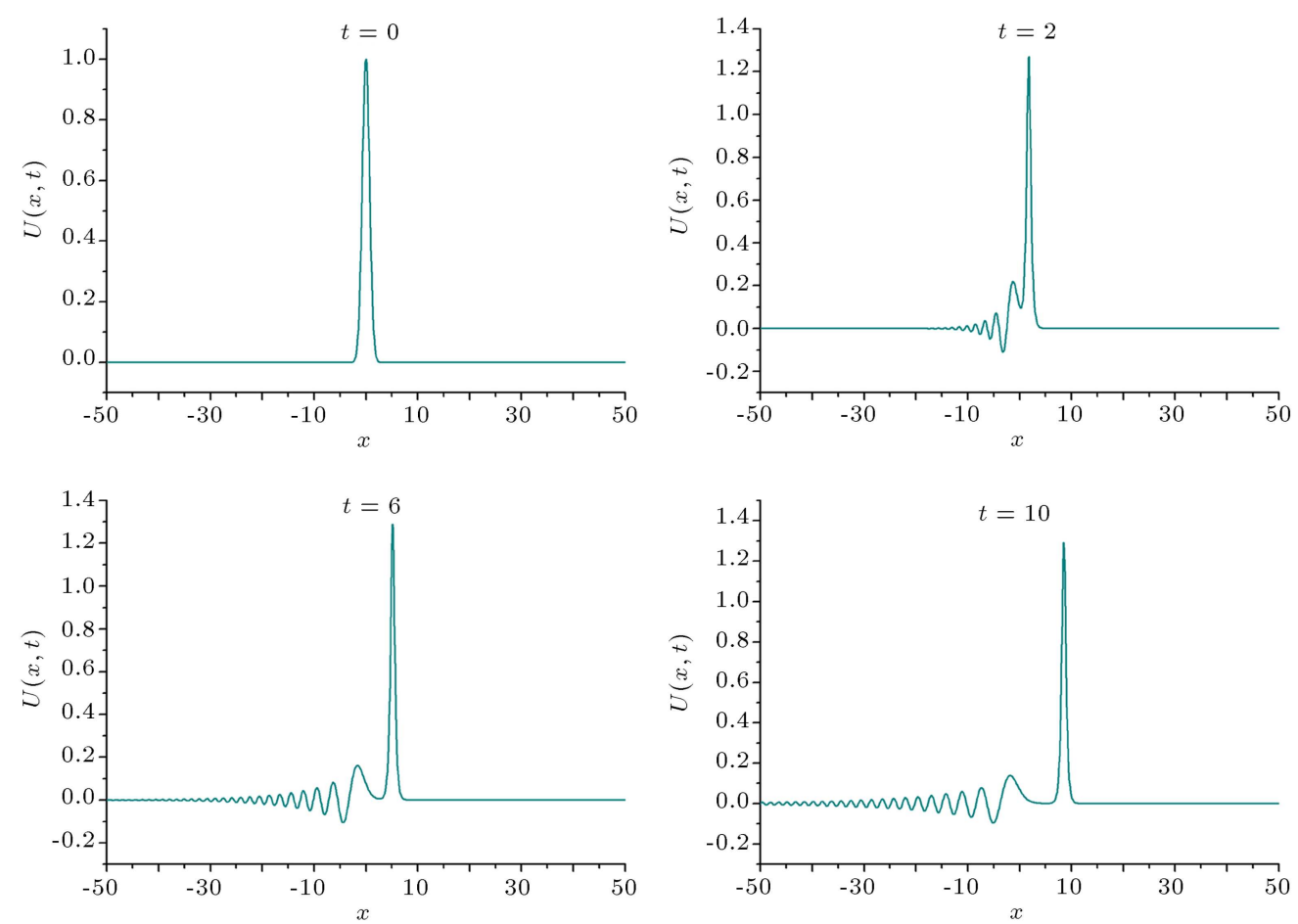

Figure 6. Gaussian initial condition with $\varepsilon=3, \mu=0.1, h=0.1, \Delta t=0.01$, and $-50 \leq x \leq 50$ at $0 \leq t \leq 10$.

equation has been introduced using cubic B-spline Petrov-Galerkin finite element method. To examine the performance of the scheme, four test problems have been studied. To show the performance of numerical scheme, the error norms $L_{2}$ and $L_{\infty}$ for single solitary wave and conserved quantities $I_{1}$ and $I_{2}$ for three test problems have been calculated. These calculations represent that the obtained error norms are smaller than the existing numerical results in the literature. The changes of the invariants are sufficiently small and the quantities of the invariants are consistent with those of [12]. Also, the linearized numerical scheme is unconditionally stable. Finally, the presented method can be reliably applied to obtain the numerical solution to the mKdV equation and similar types of non-linear problems.
The results of this paper stand on a very strong footing that is encouraged with a lot of future prospects in this avenue. Later, mKdV equation will be generalized to $\mathrm{KdV}$ equation with power law nonlinearity, so that $\mathrm{KdV}$ and $\mathrm{mKdV}$ equations will fall out as their special cases. The results of this generalized version will be disseminated elsewhere. In addition, potential $\mathrm{KdV}$ equation will also be addressed numerically using this scheme and other rich algorithms. Subsequently, the extension of $\mathrm{KdV}$ equation will be touched upon. These include Gardner's equation that is otherwise known as $\mathrm{KdV}-\mathrm{mKdV}$ equation.

In order to understand the double-layered shallow water wave dynamics, there are several proposed models. A couple of them are Bona-Chen equation as well as Gear-Grimshaw system. These vector- 

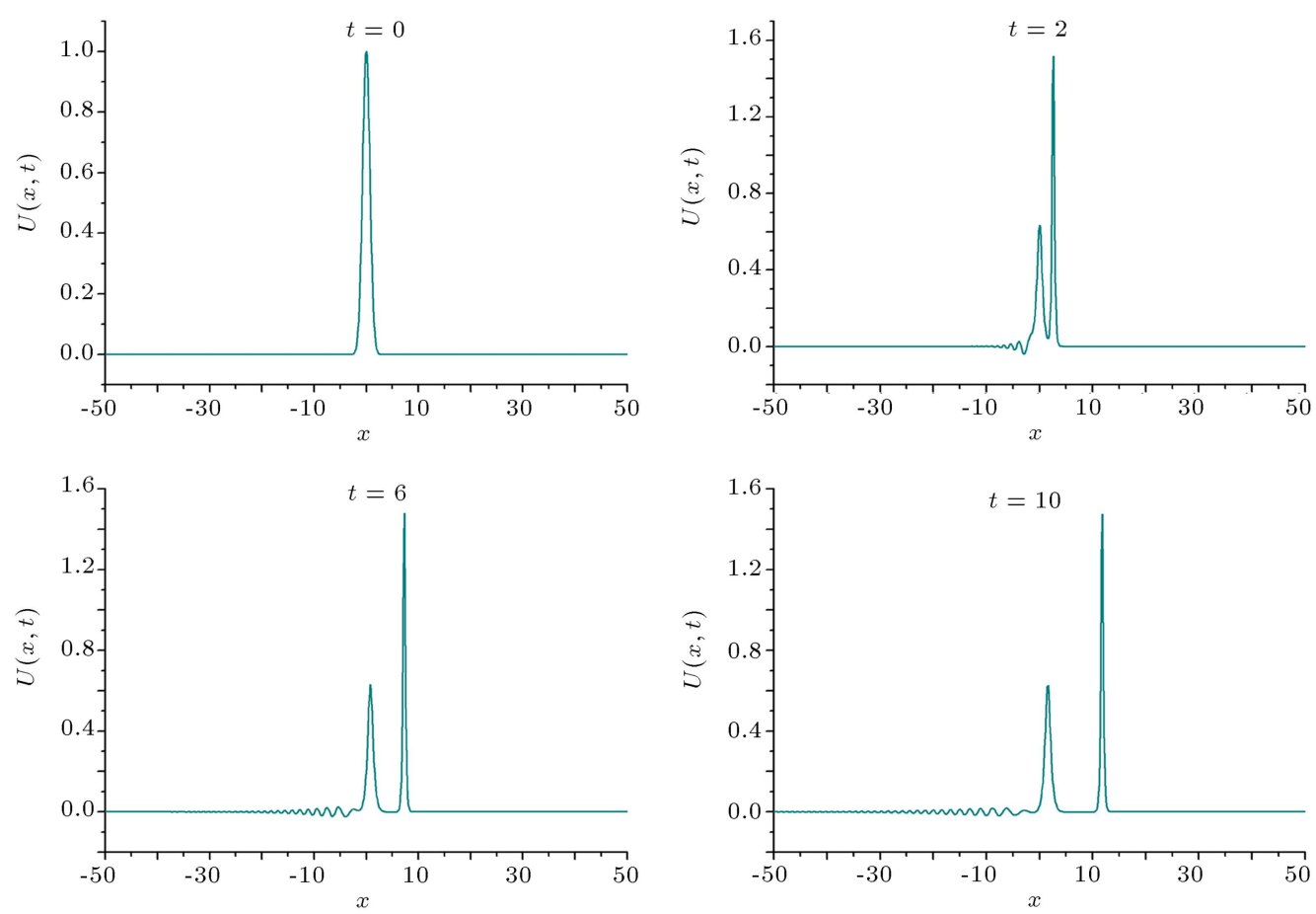

Figure 7. Gaussian initial condition with $\varepsilon=3, \mu=0.04, h=0.1, \Delta t=0.01$, and $-50 \leq x \leq 50$ at $0 \leq t \leq 10$.

coupled models will also be illustrated numerically to gain a better understanding of the dynamics of Exxon-Valdez oil spill in Alaska. The results of such research activities will be gradually and sequentially reported.

\section{Acknowledgment}

The author, Turgut Ak, is grateful to the Scientific and Technological Research Council of Turkey for granting scholarship for Ph.D. studies.

\section{References}

1. Korteweg, D.J. and de Vries, G. "On the change of form of long waves advancing in a rectangular canal, and on a new type of long stationary wave", Philosophical Magazine, 39, pp. 422-443 (1895).

2. Daeichi, M. and Ahmadian, M.T. "Application of variational iteration method to large vibration analysis of slenderness beams considering mid-plane stretching", Scientia Iranica B, 22(5), pp. 1911-1917 (2015).

3. Islam, S., Haq, S. and Ali, A. "Meshfree method for the numerical solution of RLW equations", Journal of Computational and Applied Mathematics, 223, pp. 997-1012 (2009).

4. Karakoc, S.B.G., Zeybek, H. and Ak, T. "Numerical solutions of the Kawahara equation by the septic Bspline collocation method", Statistics, Optimization and Information Computing, 2, pp. 211-221 (2014).

5. Karakoc, S.B.G., Ak, T. and Zeybek, H. "An efficient approach to numerical study of the MRLW equation with B-spline collocation method", Abstract and Applied Analysis, Article ID 59640, 15 pages (2014).

6. Karakoc, S.B.G. and Ak, T. "Numerical simulation of dispersive shallow water waves with Rosenau-KdV equation", International Journal of Advances in Applied Mathematics and Mechanics, 3(3), pp. 32-40 (2016).

7. Karakoc, S.B.G. and Ak, T. "Numerical solution of Rosenau-KdV equation using subdomain finite element method", New Trends in Mathematical Sciences, 4(1), pp. 223-235 (2016).

8. Triki, H., Ak, T., Moshokoa, S. and Biswas, A. "Soliton solutions to $\mathrm{KdV}$ equation with spatio-temporal dispersion", Ocean Engineering, 114, pp. 192-203 (2016).

9. Karakoc, S.B.G. and Geyikli, T. "Petrov-Galerkin finite element method for solving the MRLW equation", Mathematical Sciences, 7(25), pp. 1-10 (2013).

10. Kaya, D. "An application for the higher order modified KdV equation by decomposition method", Communications in Nonlinear Science and Numerical Simulation, 10(6), pp. 693-702 (2005).

11. Triki, H. and Wazwaz, A.-M. "Sub-ODE method and soliton solutions for the variable-coefficient $\mathrm{mKdV}$ equation", Applied Mathematics and Computation, 214(2), pp. 370-373 (2009).

12. Kazeminia, M., Soleimani-Amiri, S. and Zahedi, S.A. "Exact and numerical solutions for nonlinear higher order modified $\mathrm{KdV}$ equations by using variational iteration method", Advanced Studies in Theoretical Physics, 4(9), pp. 437-447 (2010). 
13. Biswas, A. and Raslan, K.R. "Numerical simulation of the modified Korteweg-de Vries equation", Physics of Wave Phenomena, 19(2), pp. 142-147 (2011).

14. Johnpillai, A.G., Khalique, C.M. and Biswas, A. "Exact solutions of the mKdV equation with timedependent coefficients", Mathematical Communications, 16(2), pp. 509-518 (2011).

15. Mansour, M.B.A. "Traveling waves for a dissipative modified KdV equation", Journal of the Egyptian Mathematical Society, 20(2), pp. 134-138 (2012).

16. Wazwaz, A.-M. “(2+1)-dimensional mKdV (N) equations by the $\mathrm{mKdV}$ recursion operator: multiple soliton and multiple singular soliton solutions", Applied Mathematics and Computation, 219(5), pp. 2535-2544 (2012).

17. Wazwaz, A.-M. "A variety of $(3+1)$-dimensional $\mathrm{mKdV}$ equations derived by using the $\mathrm{mKdV}$ recursion operator", Computers and Fluids, 93(10), pp. 41-45 (2014).

18. Raslan, K.R. and Baghdady, H.A. "New algorithm for solving the modified Korteweg-de Vries (mKdV) equation", International Journal of Research and Reviews in Applied Sciences, 18(1), pp. 59-64 (2014).

19. Raslan, K.R. and Baghdady, H.A. "A finite difference scheme for the modified Korteweg-de Vries equation", General Mathematics Notes, 27(1), pp. 101-113 (2015).

20. Wazwaz, A.-M. "New (3+1)-dimensional nonlinear evolution equations with $\mathrm{mKdV}$ equation constituting its main part: multiple soliton solutions", Chaos, Solitons and Fractals, 76, pp. 93-97 (2015).

21. Islam, M.S., Khan, K. and Akbar, M.A. "An analytical method for finding exact solutions of modified Korteweg-de Vries equation", Results in Physics, 5, pp. 131-135 (2015).

22. Prenter, P.M., Splines and Variational Methods, John Wiley, New York, USA (1975).

23. Miura, R.M., Gardner, C.S. and Kruskal, M.D. "Korteweg-de Vries equation and generalizations. II. Existence of conservation laws and constants of motion", Journal of Mathematical Physics, 9(8), p. 1204 (1968).
24. Miura, R.M. "The Korteweg-de Vries equation: A survey of results", SIAM Review, 18(3), pp. 412-459 (1976).

\section{Biographies}

Turgut Ak is a graduate student in the Department of Applied Mathematics in Nevsehir Haci Bektas Veli University, Turkey. He earned his BSc degree in Mathematics from Gazi University, Turkey. Subsequently, he obtained his MSc degree in Applied Mathematics from Erciyes University, Turkey. Currently, he is pursuing his doctoral studies. In addition, he is a Lecturer of Mathematics in the Department of Transportation Engineering at Yalova University, Turkey. His current research area of interest is numerical methods.

Seydi Battal Gazi Karakoc earned his BEd degree in Mathematics from Selcuk University, Turkey. Subsequently, he obtained his $\mathrm{MSc}$ and $\mathrm{PhD}$ degrees in Applied Mathematics from Inonu University, Turkey. Currently, he is an Assistant Professor of Mathematics in the Department of Mathematics at Nevsehir Haci Bektas Veli University, Turkey. His current research area of interest is numerical methods.

Anjan Biswas earned his BSc (Honors) degree from Saint Xaviers College in Calcutta, India. Subsequently, he received his MSc and M Phil degrees in Applied Mathematics from University of Calcutta. Then, he earned MA and PhD degrees in Applied Mathematics from the University of New Mexico in Albuquerque, New Mexico, USA. After that, he carried out his post-doctoral studies in Applied Mathematics from the University of Colorado, Boulder, USA. He was then an Assistant Professor of Mathematics at Tennessee State University that is located in Nashville, TN. Currently, he is working as an Associate Professor of Mathematics at Delaware State University in Dover, DE. His research interest is on theory of solitons. 